\title{
Avaliação in vitro da lioequivalência de formulações farmacêuticas
}

\author{
Paulo Jorge Cardoso da Costa*
}

Serviço de Tecnologia Farmacêutica, Faculdade de Farmácia da Universidade do Porto

*Correspondência:

P.J.C. Costa

Serviço de Tecnologia Farmacêutica Faculdade de Farmácia - Universidade do Porto

Rua Aníbal Cunha, 164 4050-047 - Porto - Portugal

E-mail: pccosta@ff.up.pt
A avaliação e a comparação da liberação dos fármacos a partir das formas farmacêuticas têm preocupado a indústria farmacêutica e as autoridades de registro. Utilizam-se, hoje em dia, diversos métodos para ajudar a decidir se diferentes formulações liberam os seus fármacos de modo semelhante, nomeadamente métodos estatísticos, métodos dependentes de modelos matemáticos e métodos independentes de modelos. Os métodos estatísticos utilizados podem ser baseados em testes não paramétricos, como o teste de Mann-Whitney, teste de Kolmogorov-Smirnov Z ou teste do Qui quadrado, ou testes paramétricos, como a análise de variância univariada (ANOVA) ou multivariada (MANOVA), ou o teste de t-student. A liberação do fármaco a partir de formas farmacêuticas sólidas tem sido descrita por vários modelos cinéticos, em que a quantidade de fármaco liberado (Q) é função do tempo decorrido de ensaio ( $t$ ), ou seja $Q=f(t)$. Outros parâmetros de liberação, como tempo de dissolução $\left(t_{x \%}\right)$, tempo de amostragem $\left(t_{x \min }\right)$, eficácia de dissolução $(E D)$, fator de diferenciação $\left(f_{1}\right)$, fator de semelhança $\left(f_{2}\right)$, indices de Rescigno $\left(x_{1}\right.$ e $\left.x_{2}\right)$, entre outros, podem também ser utilizados para caracterizar o perfil de liberação de fármacos.
Unitermos:

- Dissolução

- Perfis de dissolução

- Lioequivalência

- Modelos matemáticos

- Parâmetros de dissolução

\section{INTRODUÇÃO}

A avaliação e a comparação da liberação dos fármacos a partir das formas farmacêuticas têm preocupado a indústria farmacêutica e as autoridades de registro. Sempre que se produz ou desenvolve uma forma farmacêutica sólida, é necessário garantir que os fármacos sejam liberados de modo adequado. Utilizam-se, hoje em dia, diversos métodos para ajudar a decidir se diferentes formulações liberam os seus fármacos de modo semelhante. Para a realização destes estudos utilizam-se mais freqüentemente os perfis cumulativos de fármaco ao longo do tempo em oposição aos seus perfis diferenciais.

Alguns métodos para comparar perfis de liberação foram recentemente propostos (CDER, 1995; Shah, Poli,
1996, Poli et al., 1997; Ju, Liaw, 1997; Fassihi, Pillay, 1998; Costa, Lobo, 2001). Esses métodos podem ser classificados em várias categorias, nomeadamente:

$>$ Métodos estatísticos.

$>$ Métodos independentes de modelos.

$>$ Métodos dependentes de modelos matemáticos.

$>$ Outros parâmetros.

\section{MÉTODOS ESTATÍSTICOS}

Os métodos estatísticos utilizados (Tsong et al., 1996; Bartoszynski, et al., 2001) podem ser baseados em testes não-paramétricos, como o teste de Mann-Whitney (Bolton, 1984), o teste de Kolmogorov-Smirnov Z ou o teste do Qui quadrado) ou testes paramétricos (como a 
análise de variância, ou o teste de $t$-student) utilizando:

* Apenas um tempo de dissolução;

* Múltiplos tempo de dissolução;

* Distância entre as curvas de dissolução.

Os métodos estatísticos baseados na análise da variância podem ser, ainda, distinguidos em análise da variância simples (ANOVA) e análise da variância multivariada (MANOVA) (Barreto et al., 2000; Saranadasa, 2001). Os métodos estatísticos consistem na procura de diferenças significativas para a liberação do fármaco em cada amostra efetuada (ANOVA ou teste de t-student) ou na procura de diferenças significativas em séries de amostras (MANOVA).

A utilização de testes estatíticos separados para cada tempo de dissolução provoca, por vezes, um erro global do tipo I demasiado elevado, isto é, a probabilidade de rejeitar a hipótese nula $\left(H_{0}\right)$, quando verdadeira, toma valores que ultrapassam o aceitável, ou melhor, aceitam-se como diferentes dois perfis de dissolução realmente similares.

Os testes univariados ignoram muita informação importante contida nos dados como sejam as relações entre as variáveis, enquanto o teste multivariado incorpora toda esta informação na análise e na decisão final. Embora as diferenças entre perfis possam não ser significativas para cada tempo de dissolução separadamente, quando analisadas em conjunto poderão levar a diferenças significativas. Pequenas diferenças para cada tempo de dissolução poderão combinar-se para produzir diferença global mais significativa. Neste caso, os testes multivariados tornam-se mais potentes, i.e., existe maior probabilidade de rejeitar a $H_{0}$, quando ela é efetivamente falsa (menor risco de cometer um erro do tipo II; possibilidade de dois perfis de dissolução diferentes serem considerados similares).

As decisões que se retiram de testes univariados e de um teste multivariado podem ser diferentes e até mesmo contraditórias. Para contornar esta dificuldade pode proceder-se primeiro a um teste multivariado e, em caso de diferenças significativas, fazer-se, em seguida, testes univariados para identificar os tempos de dissolução que mais contribuem para essa decisão. Além dos métodos de análise de variância univariada (ANOVA), utilizam-se também, embora menos freqüentemente, os método de análise de variância multivariada (MANOVA) com estatística Lambda de Wilk's, traço de Pillai, raiz máxima de Roy e traço de Hoteling.

\section{MODELOS MATEMÁTICOS}

A liberação do fármaco a partir de sistemas de liberação imediata e modificada tem sido descrita por várias teorias/modelos cinéticos. Sendo $f_{t}$ uma função de $t$ (tempo) relacionada com a quantidade de fármaco liberado a partir do sistema terapêutico considerado, existem vários modelos para representar os perfis de liberação dos fármacos.

A interpretação quantitativa dos valores obtidos em ensaios de dissolução é muito facilitada pela utilização de uma equação genérica que traduza matematicamente a curva de dissolução em função de parâmetros relacionados com as formas farmacêuticas. Em casos especiais, essa equação pode ser deduzida por uma análise teórica de todo o processo, como por exemplo na cinética de ordem zero. Na maioria dos casos dos comprimidos, cápsulas, formas revestidas ou sistemas de liberação prolongada, esse fundamento teórico não existe e surgem, por vezes, equações empíricas mais adequadas.

Mas qual é o critério para escolher o "melhor modelo" para estudar o fenômeno de dissolução/liberação de fármacos? Um método comum usa o coeficiente de determinação, $R^{2}$, para avaliar o ajuste de um modelo para a função $Q(t)$. Contudo, este valor tende, geralmente, a aumentar com a adição de mais parâmetros ao modelo, independentemente do significado da variável adicionada. Para o mesmo número de parâmetros, o coeficiente de determinação pode ser usado para determinar a melhor definição analítica. Quando se comparam modelos com diferentes números de parâmetros, o coeficiente de determinação ajustado $\left(R_{\text {ajustado }}^{2}\right)$ tem maior significado:

$R_{\text {adjusted }}^{2}=1-\frac{(n-1)}{(n-p)}\left(1-R^{2}\right)$

Equação 1

onde, $n$ é o número de pontos $(M / t), p$ é o número de parâmetros no modelo. Embora o coeficiente de determinação $\left(R^{2}\right)$ aumente sempre, ou pelo menos permaneça constante quando se adiciona um novo parâmetro ao modelo, o coeficiente de determinação ajustado realmente diminui, dando assim indicação se um novo parâmetro realmente melhora o modelo ou se pelo contrário pode levar a um desajuste. A escolha do modelo que melhor explica o processo de liberação nem sempre é um processo fácil e baseia-se em algumas considerações, como, no fato dos desvios resultantes do ajustamento de cada modelo deverem estar aleatoriamente distribuídos, um valor o mais próximo de 1 para o coeficiente de correlação e a soma dos quadrados dos desvios $(S Q D)$ ser razoavelmente pequena ou se possível nula. Devido ao fato de quanto maior for o número de parâmetros do modelo utilizado, maior é a possibilidade do valor de $S Q D$ ser menor, então para a seleção da melhor definição analítica para a função $Q(t)$ utilizam-se parâmetros discriminatórios de modelos, 
sendo um dos mais utilizados o critério de informação de Akaike ("Akaike information criteria" - AIC). Quando se comparam vários modelos para analisar o mesmo conjunto de informação, o modelo associado com mais baixo valor de $A I C$ é considerado como o que permite obter o melhor ajuste do conjunto de modelos escolhidos e testados:

$$
A I C=M x \ln (S Q D A)+2 x P
$$

onde $M$ é o número de pares de valores experimentais $(Q / t), P$ é o número de parâmetros do modelo de ajuste e $S Q D A$ é a soma dos quadrados dos desvios, ajustada.

O uso de pelo menos cinco tempos de dissolução é recomendado para descrição adequada de um perfil de dissolução. Por vezes, nem todos os pontos da curva de dissolução podem ser utilizados no cálculo dos parâmetros cinéticos, devendo esses pontos ser muito bem escolhidos. O ajuste dos modelos deve ser sempre verificado, não se devendo utilizar modelos em que o coeficientes de determinação sejam baixos (geralmente $R^{2}<0,99$ corresponde a um ajuste deficiente).

\section{Cinética de Ordem Zero}

A liberação de formas farmacêuticas que não se desagreguem e que liberam o fármaco lentamente (assumindo que a área muda de um modo desprezível e que não se atingem condições de equilíbrio) pode ser expressa da seguinte maneira:

\section{$W_{0}-W_{t}=K t \quad$ Equação 3}

sendo $W_{0}$ a quantidade inicial de fármaco na forma farmacêutica, $W_{t}$ a quantidade que resta de fármaco na forma farmacêutica ao fim do tempo $t$ e $K$ uma constante de proporcionalidade. Dividindo a equação por $W_{0}$ e simplificando teremos:

$$
f_{t}=K_{0} t \quad \text { Equação } 4
$$

sendo $f_{t}=1-\left(W / W_{0}\right)$, em que $f_{t}$ representa a fração de fármaco liberado no tempo $t$ e $K_{0}$ a constante de velocidade aparente de dissolução ou constante de liberação de ordem zero. Desse modo, um gráfico da fração liberada do fármaco versus tempo será linear se as condições previamente estabelecidas forem cumpridas.

Esta relação pode ser utilizada para descrever a liberação de fármacos por vários tipos de formas farmacêuticas de liberação controlada, como é o caso de alguns dos sistemas transdérmicos, assim como de comprimidos matriciais, formas revestidas, sistemas osmóticos, entre outros (Varelas, Dixon, Carol, 1995). As formas farmacêuticas que seguem este perfil liberam a mesma quantidade de fármaco por unidade de tempo, constituindo-se como uma das melhores formas de veicular fármacos para liberação prolongada. Este modelo pode ser expresso, de um modo simples, pela seguinte relação:

$Q_{t}=Q_{0}+K_{0} t \quad$ Equação 5

sendo $Q_{t}$ a quantidade de fármaco liberada no tempo $t, Q_{0}$ a quantidade inicial de fármaco na solução (na maior parte das vezes, $Q_{0}=0$ ) e $K_{0}$ a constante de liberação de ordem zero (Figura 1).

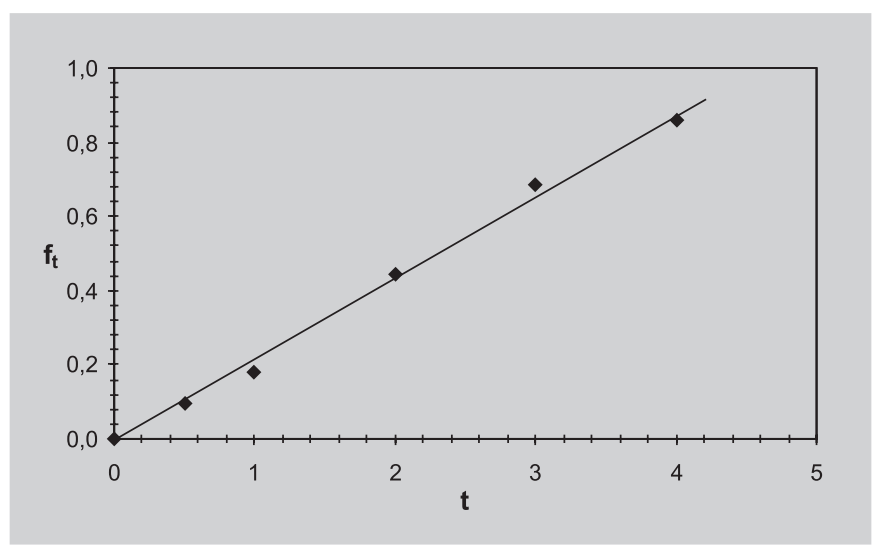

FIGURA 1 - Representação gráfica do modelo de ordem zero em que as curvas obtidas são do tipo $f(t)=a+b t$.

\section{Cinética de ordem um}

A aplicação deste modelo para o estudo da dissolução de fármacos foi proposto por Gibaldi e Felman (1967) e mais tarde, por Wagner (1969). Este modelo tem sido, também, muito empregado para descrever a absorção e/ou eliminação de alguns fármacos (Gibaldi, Perrier,1982), embora seja difícil conceituar este mecanismo em bases teóricas. Kitawaza et al. $(1995,1997)$ propuseram um modelo ligeiramente diferente, mas em que chegaram praticamente às mesmas conclusões. Este modelo pode, de um modo simples, ser expresso pela seguinte relação:

$$
\text { ou } \ln Q_{t}=\ln Q_{0}+K_{1} t \quad \text { Equação } 6
$$

ou, em logaritmos decimais:

$\log Q_{t}=\log Q_{0}+\frac{K_{1} t}{2,303}$ Equação 7 
sendo $Q_{t}$ a quantidade de fármaco liberada no tempo $t, Q_{0}$ a quantidade inicial de fármaco na solução e $K_{l}$ a constante de liberação de ordem um.

Deste modo, um gráfico do logaritmo decimal da quantidade liberada de fármaco versus o tempo decorrido de ensaio será linear. As formas farmacêuticas que seguem este perfil de liberação (Mulye, Turco, 1995), liberam uma quantidade de fármaco proporcional à quantidade restante no seu interior, por unidade de tempo, de modo que a quantidade de fármaco liberada vai diminuindo.

\section{Modelo de Weibull}

A equação empírica descrita por Weibull foi também adaptada ao processo de dissolução/liberação por Langenbucher (1972). Ela pode ser aplicada com sucesso a quase todos os tipos de curvas de dissolução e tem sido largamente utilizada nestes estudos (Goldsmith, Randall, Ross, 1978; Romero et al., 1991; Vudathala, Rogers, 1992). Este modelo é, por vezes, também conhecido como modelo de Rosin-Rammler-Sperling-Weibull (RRSW).

Quando aplicado à dissolução de fármacos a partir de formas farmacêuticas, a equação de Weibull exprime a fração acumulada de fármaco, $m$, na solução ao fím do tempo $t$ por:

$$
m=1-\exp \left[\frac{-\left(t-T_{i}\right)^{b}}{a}\right]
$$

Equação 8

Nesta equação, $a$ representa um parâmetro de escala relacionado com tempo decorrido de ensaio. O parâmetro de localização, $T_{i}$, representa o tempo de latência até que o processo de dissolução ocorra e, na maior parte dos casos, é igual a zero.

O parâmetro de forma, $b$ (Figura 2), caracteriza a curva como exponencial $(b=1)$ (Caso 1$)$, como sigmoidal, em forma de $\mathrm{S}$ com curvatura para cima, seguida de um ponto de viragem $(b>1)$ (Caso 2), ou então parabólica, com uma inclinação inicial constante, sendo depois concordante com a exponencial $(b<1)$ (Caso 3 ). Esta equação pode ser transformada em:

$$
\log [-\ln (1-m)]=b \log \left(t-T_{i}\right)-\log a \quad \text { Equação } 9
$$

Para sistemas farmacêuticos que sigam este modelo, o gráfico logaritmo decimal da quantidade liberada do fármaco versus o logaritmo decimal do tempo será linear (Figura 3).

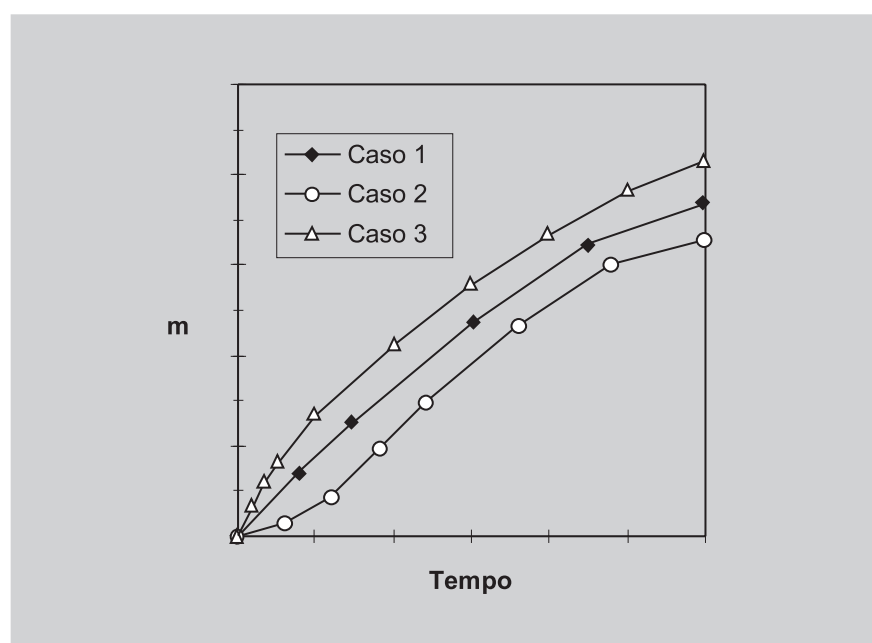

FIGURA 2 - Tipos de perfil de liberação caracterizados pelo parâmetro de forma $b$.

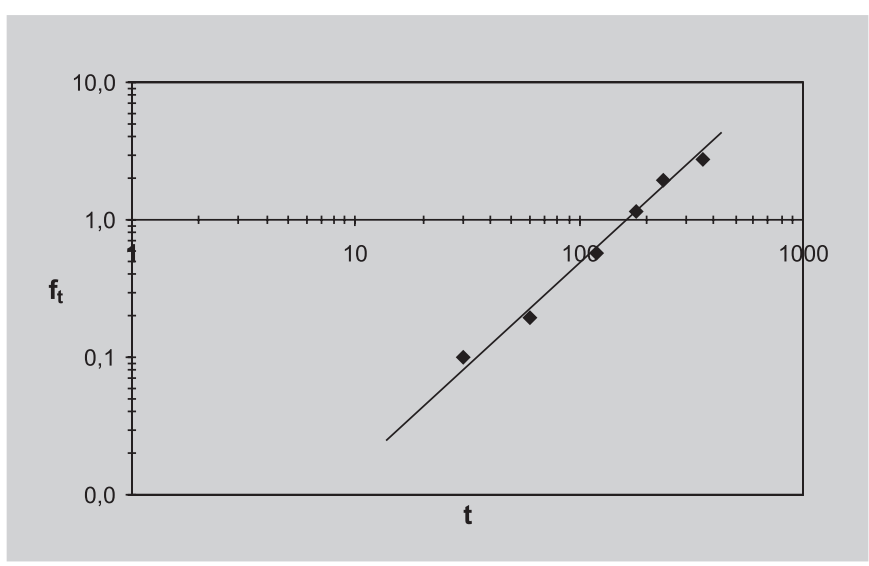

FIGURA 3 - Representação gráfica do Modelo de Weibull.

Por este ser um modelo empírico, apresenta algumas deficiências e tem sido alvo de algumas críticas (Pedersen, Myrick, 1978; Christensen, Hansen, Bechgaard, 1980), tais como não ter qualquer fundamento cinético e poder apenas descrever, mas não caracterizar adequadamente, as propriedades cinéticas de dissolução de um fármaco, não conter um parâmetro que dê uma idéia da velocidade de dissolução intrínseca do fármaco e não ajudar a estabelecer correlações in vivo/in vitro.

\section{Modelo de Higuchi}

Takeru Higuchi (Higuchi, 1961; Higuchi, 1963) desenvolveu teoricamente vários modelos para a liberação de fármacos hidrossolúveis e pouco solúveis incorporados em 
matrizes semi-sólidas e/ou sólidas. Foram obtidas relações matemáticas para partículas do fármaco homogeneamente dispersas em matriz uniforme, que se comporta como meio de difusão. Para a liberação a partir de um sistema matricial homogêneo plano, a relação obtida foi a seguinte:

$$
f_{t}=Q=\sqrt{D\left(2 C-C_{s}\right) C_{s} t} \quad \text { Equação } 10
$$

sendo $Q$ a quantidade de fármaco cedida no tempo $t$ por unidade de superfície, $C$ a concentração inicial de fármaco, $C_{S}$ a solubilidade do fármaco na matriz/excipiente e $D$ a constante de difusão das moléculas do fármaco no excipiente. Esta relação foi primeiramente proposta por Higuchi para descrever a liberação de fármacos em suspensão a partir de pomadas, mas está perfeitamente de acordo com outros tipos de liberação de outras formas farmacêuticas.

Outros modelos foram, também, desenvolvidos por Higuchi, nomeadamente para a liberação a partir de sistemas matriciais homogêneos esféricos, sistemas matriciais granulosos planos e sistemas matriciais granulosos esféricos. De modo geral, podemos resumir o modelo de Higuchi à expressão:

$$
f_{t}=K_{H} t^{1 / 2}
$$

Equação 11

sendo $K_{H}$ a constante de liberação de Higuchi, tratada por vezes de modo diferente por diferentes autores e teorias. Higuchi descreve, assim, a liberação do fármaco como um processo de difusão baseado na lei de Fick, dependente da raiz quadrada do tempo. Desse modo um gráfico da quantidade liberada do fármaco versus a raiz quadrada do tempo será linear (Figura 4) se as condições previamente estabelecidas forem cumpridas.

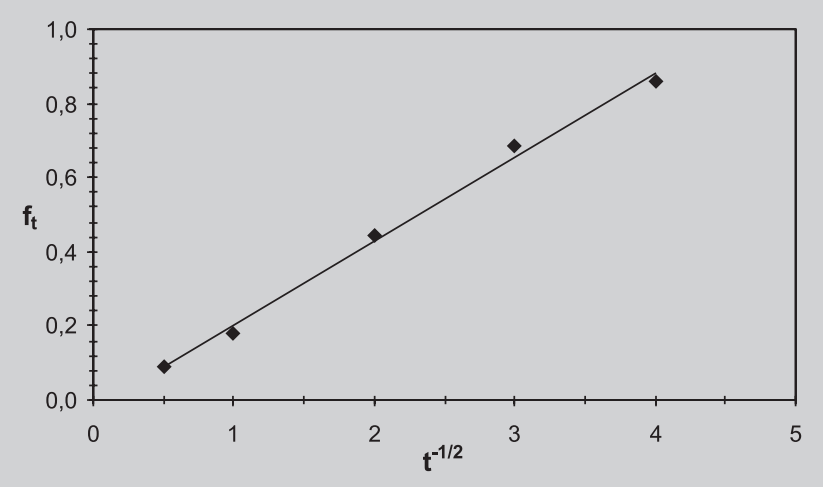

FIGURA 4 - Representação gráfica do modelo de Higuchi em que as curvas obtidas são do tipo $f(t)=a+b \times \sqrt{ } t$ ou, de outro modo $f(t)=a+b t^{0,5}$.
Esta relação tem sido muito utilizada para descrever a liberação de fármacos por vários tipos de formas farmacêuticas de liberação controlada (Desai et al., 1966a; Desai et al., 1966 b; Schwartz, Simonelli, Higuchi, 1968a; Schwartz, Simonelli, Higuchi, 1968b; Costa, Ferreira, Lobo, 1996).

\section{Modelo de Hixson-Crowell}

Hixson e Crowell (1931), reconhecendo que a área de uma partícula regular é proporcional à raiz cúbica do seu volume, derivaram uma equação que pode ser descrita da seguinte forma:

$$
W_{0}^{1 / 3}-W_{t}^{1 / 3}=K_{s} t \quad \text { Equação } 12
$$

sendo $W_{0}$ a quantidade inicial de fármaco na forma farmacêutica, $W_{t}$ a quantidade que resta de fármaco na forma farmacêutica ao fim do tempo $t$ e $K_{s}$ uma constante que incorpora a relação superfície/volume. Esta relação aplica-se à forma farmacêutica comprimido, em que a dissolução decorre em planos paralelos à superfície de exposição se as dimensões do comprimido diminuirem proporcionalmente, de modo que a geometria inicial se mantenha durante todo o tempo. Dividindo esta equação por $W_{0}^{1 / 3}$ e simplificando teremos:

$$
\left(1-f_{t}\right)^{1 / 3}=1-K_{\beta} t
$$

Equação 13

sendo $f_{t}=1-\left(W / W_{0}\right)$, em que $f_{t}$ representa a fração de fármaco liberado ao fim do tempo $t$ e $K_{b}$ é a constante de liberação. Assim, um gráfico da raiz cúbica da fração não liberada do fármaco versus tempo será linear se as condições de equilíbrio não forem atingidas e se a forma geométrica da forma farmacêutica diminuir proporcionalmente ao longo do tempo. Quando se usa este modelo, assumese que a velocidade de liberação é limitada pela velocidade de dissolução das partículas do fármaco e não pela difusão que possa ocorrer através da matriz polimérica.

Este modelo tem sido usado para descrever o perfil de liberação tendo em conta a diminuição da superfície das partículas de fármaco à medida que a dissolução ocorre (Niebergall, Milosovich, Goyan, 1963; Prista, Alves, Morgado, 1995).

\section{Modelo de Korsmeyer-Peppas}

Este modelo foi desenvolvido por Korsmeyer e Peppas (Korsmeyer, Peppas, 1981; Korsmeyer et al., 1983) e pode ser representado pela seguinte expressão: 


$$
\frac{M_{t}}{M_{\infty}}=a t^{n}
$$

Equação 14

em que $a$ representa uma constante cinética que incorpora as características estruturais e geométricas da forma de liberação controlada, $n$ é o chamado expoente de liberação para a liberação do fármaco e $M / M_{\infty}$ é a fração do fármaco liberada ao longo do tempo $t$.

Peppas (1985) usou o valor de $n$ de modo a caracterizar os diferentes mecanismos de liberação, tendo chegado a valores de $n=0,5$ para a difusão (modelo de Fick) e valores de $n$ mais elevados entre 0,5 e 1,0 num sistema plano, para a transferência de massa segundo um modelo não-Fickiano (Tabela I). No caso de cilindros (comprimidos) $n$ é 0,45 em vez de 0,5 e 0,89 em vez de 1,0 .

Este modelo é geralmente usado para analisar a liberação de formas farmacêuticas poliméricas, quando o mecanismo de liberação não é bem conhecido ou quando possa estar envolvido mais de um tipo de liberação.

Uma equação modificada tem sido utilizada (Ford et al., 1987; Harland et al., 1988; Ford et al., 1991; Kim, Fassihi, 1997) de modo a incorporar o tempo de latência ( $l$ no início da liberação do fármaco a partir da forma farmacêutica:

$$
\frac{M_{(t-l)}}{M_{\infty}}=a(t-l)^{n}
$$

ou, a sua versão logarítmica:

$$
\log \left(\frac{M_{(t-l)}}{M_{\infty}}\right)=\log a+n \log (t-l) \quad \text { Equação } 16
$$

Quando há a possibilidade de uma liberação inicial rápida ("burst effect"), $b$, esta equação transforma-se em (Ford et al., 1991):

$$
\frac{M_{t}}{M_{\infty}}=a t^{n}+b \quad \text { Equação } 17
$$

O significado e o mecanismo desta liberação inicial rápida não foram ainda completamente esclarecidos e nenhuma teoria que descreva este fenômeno foi até agora desenvolvida com sucesso (Huang, Brazel, 2001). Na ausência de um tempo de latência ou uma liberação inicial rápida, os valores de $l$ e $b$ tornam-se zero e apenas $a t^{n}$, na parte direita da equação, é usado. Esta relação foi utilizada para descrever a liberação de fármacos por vários tipos de formas farmacêuticas de liberação controlada (Ford et al., 1991; Sangalli et al., 1994; Lin, Yang, 1989).

\section{Modelo de Hopfenberg}

A liberação de fármacos de sistemas com diversas geometrias em cuja superfície ocorre erosão foi objeto de estudo por Hopfenberg (1976), e mais tarde por Katzhendler et al. (1997), que propôs a seguinte equação para matrizes planas, esféricas e cilíndricas apresentando erosão heterogênea:

$$
\frac{M_{t}}{M_{\infty}}=1-\left[1-\frac{k_{0} t}{C_{0} a_{0}}\right]^{n} \quad \text { Equação } 18
$$

em que $M_{t}$ é a quantidade de fármaco liberada durante o tempo $t$, e $M_{\infty}$ é a quantidade total de fármaco disponível para liberação (quando se atinge a exaustão do sistema), sendo assim $M / M_{\infty}$ a fração do fármaco liberada. $k_{0}$ é a constante de erosão, $C_{0}$ é a concentração inicial do fármaco na matriz e $a_{0}$ é o raio inicial para esferas e cilindros e metade da espessura para sistemas planos. O valor de $n$ é 1,2 e 3 para sistemas planos, cilíndricos e esféricos, respectivamente. Uma forma modificada deste modelo foi desenvolvido (El-Arini, Leuenberger, 1998) para acomodar o tempo de latência $(l)$, que surge, por vezes, no início da liberação do fármaco a partir da forma farmacêutica:

$$
\frac{M_{t}}{M_{\infty}}=1-\left[1-k_{1} t(t-l)\right]^{n} \quad \text { Equação } 19
$$

TABELA I - Interpretação dos mecanismos de liberação a partir de películas poliméricas

\begin{tabular}{ccc}
\hline Expoente de liberação $(n)$ & Mecanismo de transporte do fármaco & Velocidade como função do tempo \\
\hline 0,5 & Difusão (de acordo com a lei de Fick) & $\mathrm{t}^{-0,5}$ \\
entre 0,5 e 1,0 & Transporte anómalo & $\mathrm{t}^{\mathrm{n}-1}$ \\
1,0 & Transporte Caso-II & Ordem zero \\
superior a 1,0 & Super Transporte Caso-II & $\mathrm{t}^{\mathrm{n}-1}$ \\
\hline
\end{tabular}


onde $k_{l}$ é igual a $k_{0} C_{0} a_{0}$. Este modelo assume que a liberação não é influenciada pela difusão interna ou externa à matriz, mas sim que o processo limitante é a própria erosão.

\section{Modelo de Baker-Lonsdale}

Este modelo foi desenvolvido por Baker e Lonsdale (1974), a partir do modelo de Higuchi e descreve a liberação controlada a partir de um matriz esférica, sendo representado pela seguinte expressão:

$$
\frac{3}{2}\left[1-\left(1-\frac{M_{t}}{M_{\infty}}\right)^{2 / 3}\right]-\frac{M_{t}}{M_{\infty}}=k t
$$

onde $M_{t} / M_{\infty}$ é a fração do fármaco liberada e $k$ a constante de liberação.

Deste modo, um gráfico relacionando a parte esquerda da equação e o tempo será linear se as condições estabelecidas forem cumpridas, a constante de liberação $(k)$ correspondendo à inclinação da reta. Esta equação tem sido usada para linearizar resultados de ensaios de liberação de várias formulações de micro-sferas ou microcápsulas (Jun, Lai, 1983; Shukla, Price, 1989; Seki et al., 1980; Shukla, Price, 1991; Bhanja, Pal, 1994).

\section{Modelo de Makoid-Banakar}

Esta equação empírica foi desenvolvida por Makoid e Banakar para simular e modelar o perfil de dissolução desejado de um fármaco antes da sua real formulação. Quando aplicado à liberação de fármacos a partir de formas farmacêuticas (Pais, 2001), este modelo expressa a fração acumulada de fármaco na solução, $M_{t} M_{\infty}$, no tempo $t$ pela seguinte expressão:

$$
\frac{M_{t}}{M_{\infty}}=c t^{n} e^{(-k t)}
$$

onde $c, n$ e $k$ são meros parâmetros de ajuste $(c, n, k>0)$.

\section{MÉTODOS INDEPENDENTES DE MODELOS}

Entre os métodos independentes de modelos podemos, ainda, encontrar os testes relacionais e os procedimentos emparelhados. Os testes relacionais são razões entre grandezas obtidas do ensaio de liberação do produto de referência e do ensaio de liberação do produto em comparação no mesmo tempo e vão desde a relação das percentagens de fármaco dissolvido $\left(t_{x^{\circ} \%}\right)$ à relação das áreas sob a curva de liberação $(A S C)$ ou à relação entre os tempos médios de dissolução $(T M D)$.

Os procedimentos emparelhados incluem os índices de Rescigno (Rescigno, 1992), o fator de diferenciação e o fator de semelhança (Moore, Flanner, 1996). Num trabalho recente, outro método independente de modelos foi proposto, baseado no valor absoluto do logaritmo da razão das áreas sob a curva de liberação de ambas as formulações testadas (Gohel, Panchal, 2000).

O fator de diferenciação $\left(f_{l}\right)$ calcula a diferença percentual entre as duas curvas a cada tempo de leitura e é uma medida do erro relativo entre as duas curvas:

$$
f_{l}=\frac{\sum_{j=1}^{n}\left|R_{j}-T_{j}\right|}{\sum_{j=1}^{n} R_{j}} \times 100
$$

Equação 22

onde $n$ é o número de recolhas, $R$ é a quantidade dissolvida do produto de referência no tempo $j$ e $T$ é a quantidade dissolvida do produto em estudo no mesmo tempo.

O fator de semelhança $\left(f_{2}\right)$ como definido pela FDA e pela EMEA é o logaritmo do recíproco da raiz quadrada de um mais o quadrado médio da diferença da percentagem de fármaco dissolvida entre os produtos teste e de referência:

$$
\begin{array}{r}
f_{2}=50 \times \log \left\{\left[1 / \sqrt{1+\frac{1}{n} \sum_{j=1}^{n}\left(R_{j}-T_{j}\right)^{2}}\right] \times 100\right\} \\
\text { Equação } 23
\end{array}
$$

Este método é mais adequado para a comparação de perfis de liberação quando estão disponíveis mais de três ou quatro tempos de dissolução (no mínimo três, em que não mais de um ponto ultrapasse $85 \%$ do teor inicial). $\mathrm{O}$ fator de semelhança foi, entretanto, adotado pelo "Center for Drug Evaluation and Research" (CDER, 1995) e pela "Human Medicines Evaluation Unit of The European Agency for the Evaluation of Medicinal Products" (EMEA, 1999) para ser utilizado como medida de comparação entre perfis de liberação.

De modo a considerar os perfis de dissolução como similares, o valor de $f_{1}$ deve ser próximo de 0 e o valor de $f_{2}$ deve ser próximo de 100 . De um modo geral, valores de $f_{1}$ menores que $15(0-15)$ e valores de $f$ maiores que 50 (50-100) mostram a semelhança dos perfis de dissolução. A FDA e a EMEA sugerem que dois perfis de dissolução sejam declarados similares se o valor de $f_{2}$ estiver entre 50 
e 100. Em adição, ele requer que se utilizem pelo menos 12 unidades individuais para usar o fator de semelhança para comparar a dissolução. Os perfis deverão ser obtidos nas mesmas condições de ensaio (mesmos tempos de coleta, equipamento e velocidade de agitação). Uma vez que este fator não considera a dispersão das respostas obtidas a cada tempo de resposta, não se devem utilizar perfis médios obtidos com variabilidade superior a $20 \%$ para os primeiros tempos (até 15 minutos) e superiores a $10 \%$ para os restantes tempos.

Alguns problemas estatísticos relativos ao fator de semelhança foram apresentados por Liu e Chow (1996) e Liu, Ma e Chow (1997). Contudo, alguns procedimentos tendo em vista corrigir ou eliminar estas deficiências foram propostos por Shah et al. (1998), Reppas e Nicolaides (2000) e Costa (2001).

O novo fator de semelhança, $S_{d}$, expressa a diferença percentual entre dois perfis de dissolução. Ele é definido como a razão entre a percentagem média de fármaco dissolvido e o tempo médio de dissolução (Gohel, Panchal, 2000):

$$
S_{d}=\frac{\sum_{t=1}^{n-1}\left[\log \left(\frac{A S C_{R_{t}}}{A S C_{T_{t}}}\right)\right]}{n-1}
$$

Equação 24

onde $n$ é o número de amostras efetuadas durante o teste de dissolução e $\left[A S C_{R t}\right]$ e $\left[A S C_{T t}\right]$ são as áreas sob a curva de dissolução da formulação de referência e teste, respectivamente, no tempo $t$.

Outro método independente de modelos, foi sugerido por Tsong et al. (1996), com base no uso da distância de Mahalanobis (distância-M) entre dois vetores de variáveis ocasionais distribuídos normalmente:

$$
D_{M}=\sqrt{\left[\left(x_{2}-x_{1}\right)^{\prime} S_{\text {pooled }}^{-1}\left(x_{2}-x_{1}\right)\right]} \quad \text { Equação } 25
$$

onde $S_{\text {pooled }}=\left(S_{1}+S_{2}\right) / 2$ é a matriz variância-covariância calculada entre as duas formulações, $x_{1}=\left(x_{11}, x_{12}, \ldots, x_{1 p}\right)$ é a dissolução média da formulação de referência e $x_{2}$ é a dissolução média da formulação testada.

Estes parâmetros são utilizados para comparar perfis de liberação de duas formulações, sendo necessário considerar uma delas como formulação de referência, o que em alguns países se torna de todo impossível. Para o fator de diferenciação, o mesmo par de formulações farmacêuticas pode apresentar valores de $f_{l}$, diferentes consoante a formulação escolhida para referência. Uma adaptação da fórmula proposta por Costa (1999), usada para calcular o fator de diferenciação $\left(f_{1}^{\prime}\right)$, poderia contornar este problema:

$$
f_{l}^{\prime}=\frac{\sum_{j=1}^{n}\left|R_{j}-T_{j}\right|}{\sum_{j=1}^{n}\left(R_{j}+T_{j}\right) / 2} \times 100
$$

em que se usaria como divisor não apenas o somatório dos valores da formulação de referência, mas sim a média dos somatórios dos valores das duas formulações.

O índice de Rescigno $\left(x_{i}\right)$ foi primeiramente introduzido como um método para comparar perfis de concentrações plasmáticas de fármaco ao longo do tempo:

$$
\xi_{i}=\left(\frac{\sum_{j=1}^{n} w_{j}\left|R_{j}-T_{j}\right|^{i}}{\sum_{j=1}^{n} w_{j}\left|R_{j}+T_{j}\right|^{i}}\right)^{1 / i}
$$

Equação 45

onde $R_{j}$ é a quantidade dissolvida do produto de referência, $T_{j}$ é a quantidade dissolvida do produto em comparação no mesmo tempo $j, i$ é um número inteiro positivo, $n$ é o número total de recolhas e $w_{j}$ é um fator, opcional, que reflete o peso a dar a cada amostra (como no fator de semelhança).

Este índice, adimensional, apresenta sempre valores entre 0 e 1 inclusive e mede a diferença entre os dois perfis de liberação. Assim, apresenta o valor de 0 quando as duas curvas de liberação são idênticas (as duas formulações são perfeitamente equivalentes) e o valor de 1 quando um dos perfis é zero, ou seja, quando não há liberação. São geralmente calculados dois índice de Rescigno $x_{1}$, substituindo na fórmula $i$ por 1 , ou $x_{2}$, em que $i=2$.

\section{OUTROS PARÂMETROS DE LIBERAÇÃO}

Outros parâmetros utilizados para, de algum modo, caracterizar o perfil de liberação são o $t_{x \%}$, o tempo de amostragem e a eficácia de dissolução. $\mathrm{O} t_{x \%}$ corresponde ao tempo necessário para se liberar determinada percentagem de fármaco (ex. $t_{20 \%}, t_{50 \%}, t_{80 \%}$ ) e o tempo de amostragem corresponde ao teor de fármaco liberado nesse tempo (ex. $t_{20 \text { min }}, t_{50 \text { min }}, t_{90 \text { min }}$ ). As Farmacopéias utilizam freqüentemente este parâmetro como limite de aceitação do ensaio de dissolução (ex. $t_{45 \min }^{3} 80 \%$ ).

A eficácia de dissolução (E.D.) de uma forma farmacêutica (Khan, Rhodes, 1972; Khan, 1975) está representada na Figura 5 e pode ser calculada pela seguinte equação: 


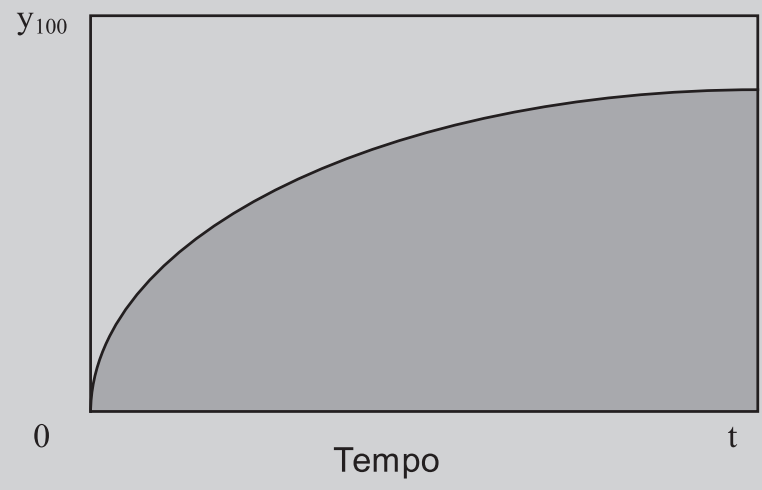

E.D. $(\%)=\frac{A S}{R} \times 100$

$A S$ - Área Sombreada

$R$ - Área do Retângulo $\left(y_{100} \times t\right)$

FIGURA 5 - Eficácia de dissolução de um fármaco a partir de uma forma farmacêutica.

$$
\text { E.D. }=\frac{\int_{0}^{t} y \times d t}{y_{100} \times t} \times 100 \%
$$

Equação 26

onde $y$ é a percentagem de fármaco liberado e $t$ é o tempo decorrido de ensaio.

Estes parâmetros contribuem com pouca informação para o esclarecimento do mecanismo de liberação e devem ser usados associados entre si ou com alguns dos modelos referidos anteriormente.

\section{CONCLUSÕES}

Como foi anteriormente referido, a interpretação quantitativa dos valores obtidos em ensaios de dissolução é muito facilitada pela utilização de equações matemáticas que descrevam a curva de liberação em função de parâmetros relacionados com as formas farmacêuticas. Alguns dos mais significativos e mais utilizados modelos matemáticos que descrevem a curva de dissolução estão referidos na Tabela II.

$\mathrm{O}$ transporte dos fármacos no interior dos sistemas farmacêuticos e a sua liberação envolvem, por vezes, múltiplas etapas provocadas por diferentes fenômenos físicos ou químicos, tornando-se, neste caso, difícil, senão mesmo impossível, arranjar um modelo matemático que descreva de modo correto todo esse conjunto complexo de acontecimentos. Os modelos descrevem melhor a liberação dos fármacos a partir dos sistemas farmacêuticos quando ela resulta de um fenômeno simples ou quando esse fenômeno, pelo fato de ser a etapa limitante, condiciona todo o processo. Estes modelos de liberação também podem ser encarados de um ponto de vista diferen-
TABELA II - Modelos matemáticos mais utilizados para descrever a curva de dissolução

\begin{tabular}{|c|c|}
\hline Ordem zero & $Q_{t}=Q_{0}+K_{0} t$ \\
\hline Ordem um & $\ln Q_{t}=\ln Q_{0}+K_{l} t$ \\
\hline Ordem dois & $\mathrm{Q}_{\mathrm{t}} / \mathrm{Q}_{\infty}\left(\mathrm{Q}_{\infty}-\mathrm{Q}_{\mathrm{t}}\right)=\mathrm{K}_{2} \mathrm{t}$ \\
\hline Hixson Crowell & $Q_{0}^{1 / 3}-Q_{t}^{l / 3}=K_{s} t$ \\
\hline Weibull & $\log \left[-\ln \left(1-\left(Q_{t} / Q_{\infty}\right)\right)\right]=b \times \log t-\log a$ \\
\hline Higuchi & $Q_{t}=K_{H} \sqrt{t}$ \\
\hline Baker-Lonsdale & $(3 / 2)\left[1-\left(1-\left(Q_{t} / Q_{\infty}\right)\right)^{2 / 3}\right]-\left(Q_{t} / Q_{\infty}\right)=K t$ \\
\hline Korsmeyer-Peppas & $Q_{t} / Q_{\infty}=K_{k} t^{n}$ \\
\hline Makoid-Banakar & $Q_{t} / Q_{\infty}=c t^{n} e^{(-k t)}$ \\
\hline Quadrático & $Q_{t}=100\left(K_{1} t^{2}+K_{2} t\right)$ \\
\hline Logístico & $Q_{t}=A /\left[1+e^{-K(t-y)}\right]$ \\
\hline Gompertz & $Q_{t}=A e^{-e-K(t-y)}$ \\
\hline Hopfenberg & $Q_{t} / Q_{\infty}=1-\left[1-k_{0} t / C_{0} a_{0}\right]^{n}$ \\
\hline
\end{tabular}


te, utilizados na otimização de formulações (Levent, 2001), permitindo obter uma equação matemática que possa ajudar a selecionar e preparar uma formulação com determinadas características de liberação.

Os modelos de liberação com maior aplicação e que melhor descrevem o fenômeno da liberação são, regra geral, o modelo de Higuchi, o modelo de ordem zero, o modelo de Weibull e o modelo de Korsmeyer-Peppas. O modelo de Higuchi e modelo de ordem zero representam dois casos limites no fenômeno de transporte e liberação dos fármacos, apresentando-se o modelo de Korsmeyer-Peppas como um parâmetro de decisão entre estes dois modelos.

Para caracterizar o perfil de liberação de fármacos é também possível recorrer a parâmetros como o $t_{x^{\circ} \%}$ o tempo de amostragem (parâmetro muito utilizado pela generalidade das Farmacopéias) e a eficácia de dissolução. Como já foi referido, a informação obtida a partir destes parâmetros para o esclarecimento do mecanismo de liberação é muito limitada, devendo estes parâmetros ser usados associados entre si ou a alguns dos modelos referidos.

Os procedimentos emparelhados, como o fator de diferenciação $\left(f_{1}\right)$, o fator de semelhança $\left(f_{2}\right)$ e os índices de Rescigno $\left(x_{i}\right)$, também apresentam o mesmo problema referido anteriormente. Além disso, estes parâmetros são usados para comparar o perfil de liberação de duas formulações diferentes, sendo necessário considerar um deles como a formulação de referência. Estes procedimentos emparelhados refletem apenas a maior ou menor semelhança entre estes dois perfis e podem ser considerados como uma boa ferramenta para julgar a equivalência de dissolução.

\section{ABSTRACT \\ In vitro evaluation of the lyoequivalence of pharmaceutical formulations}

The pharmaceutical industry and the registration authorities are very interested in the evaluation and comparison of drug release from the pharmaceutical dosage forms. Nowadays several methods are used to help decide if different formulations release their drugs in a similar way, namely statistical methods, mathematical model dependent methods and model independent methods. The statistical methods can be based on nonparametric tests, such as Mann-Whitney test, Kolmogorov-Smirnov Z test or chi-squared test or on parametric tests such as the analysis of variance, univariate (ANOVA) or multivariate (MANOVA), or the $t$-student test. Drug release from solid dosage forms has been described by kinetic models in which the dissolved amount of drug $(Q)$ is a function of the test time $(t)$, or $Q=f(t)$. Other release parameters, as dissolution time $\left(t_{x \%}\right)$, assay time $\left(t_{x \min }\right)$, dissolution efficacy (ED), difference factor $\left(f_{1}\right)$, similarity factor $\left(f_{2}\right)$ and Rescigno index ( $x_{1}$ and $\left.x_{2}\right)$, can also be used to characterize the drug dissolution/release profiles.

UNITERMS: Dissolution. Dissolution profile. Lyoequivalence. Mathematical models. Dissolution parameters.

\section{REFERÊNCIAS BIBLIOGRÁFICAS}

BAKER, R. W., LONSDALE, H. S. Controlled release: mechanisms and rates. In: TAQUARY, A. C., LACEY, R. E., eds. Controlled Release of Biologically Active Agents. New York: Plenum Press, 1974. p.15-71.

BARRETO, M., ZILDA, M., BICA, A., FARINHA, A. R. Desempenho de diferentes metodologias para comparação de perfis de dissolução In Vitro- $\mathrm{f}_{1}, \mathrm{f}_{2} \mathrm{e}$ Anova. Rev. Port. Farm., Lisboa, v.50, p.171-176, 2000.

BARTOSZYNSKI, R., POWERS, J. D., HERDERICK, E. E., PULTZ, J. A. Statistical comparison of dissolution curves. Pharmacol. Res., London, v.43, p.369-387, 2001.

BHANJA, R. S., PAL, T. K. In-vitro release kinetics of salbutamol sulphate microcapsules coated with both Eudragit RS 100 and Eudragit RL 100. Drug Dev. Ind. Pharm., New York, v.20, p.375-386, 1994.

BOLTON, S. Nonparametric methods. In: Pharmaceutical Statistics. Practical and Clinical Applications. Drugs and the Pharmaceutical Sciences. New York: Marcel Dekker, Inc., 1984. V. 26, p.396-399.

CHRISTENSEN, F. N., HANSEN, F. Y., BECHGAARD, H. Physical interpretation of parameters in the RosinRammler-Sperling-Weibull distribution for drug release from controlled release dosage forms. J. Pharm. Pharmacol., London, v.32, p.580-582, 1980.

COSTA, P. An alternative method to the evaluation of similarity factor in dissolution testing. Int. J. Pharm., Amsterdam, v.220, p.77-83, 2001

COSTA, P., FERREIRA, D. C., SOUSA LOBO, J. M. Nitroglicerina em sistemas de libertação transdérmica determinação da velocidade de libertação. Rev. Port. Farm., Lisboa, v.46, p.4-8, 1996. 
COSTA, P. Formas farmacêuticas sólidas. Estudo comparativo de cinéticas de libertação. Porto, 1999. 261p. [Tese de Doutoramento. Faculdade de Farmácia da Universidade do Porto].

COSTA, P., SOUSA LOBO, J. M. Modeling and comparison of dissolution profiles. Eur. J. Pharm. Sci., Amsterdam, v.13, p.123-133, 2001.

DESAI, S. J., SINGH, P., SIMONELLI, A. P., HIGUCHI, W. I. Investigation of factors influencing release of solid drug dispersed in inert matrices III. Quantitative studies involving the polyethylene plastic matrix.J. Pharm. Sci., New York, v.55, p.1230-1234, 1966.

DESAI, S. J., SINGH, P., SIMONELLI, A. P., HIGUCHI, W. I. Investigation of factors influencing release of solid drug dispersed in inert matrices IV. Some studies involving the polyvinyl chloride matrix. J. Pharm. Sci., New York, v.55, p.1235-1239, 1966.

EL-ARINI, S. K., LEUENBERGER, H. Dissolution properties of praziquantel-PVP systems. Pharm. Acta Helv., Amsterdam, v.73, p.89-94, 1998.

FASSIHI, R., PILLAY, V. Evaluation and comparison of dissolution data derived from different modified release dosage forms: an alternative method. J. Control. Release, Amsterdam, v.55, p.45-55, 1998.

FORD, J. L., MITCHELL, K., ROWE, P., ARMSTRONG, D. J., ELLIOTT, P. N. C., ROSTRON, C., HOGAN, J. E. Mathematical modelling of drug release from hydroxypropylmethylcellulose matrices: Effect of temperature. Int. J. Pharm., Amsterdam, v.71, p.95-104, 1991.

FORD, J .L., RUBINSTEIN, M. H., MCCAUL, F., HOGAN, J. E., EDGAR, J. E. Importance of drug type, tablet shape and added diluent on drug release kinetics from HPMC matrix tablets. Int. J. Pharm., Amsterdam, v.40, p.223-234, 1987.

GIBALDI, M., FELDMAN, S. Establishment of sink conditions in dissolution rate determinations - theoretical considerations and application to nondisintegrating dosage forms. J. Pharm. Sci., New York, v.56, p.12381242, 1967.

GIBALDI, M., PERRIER, D. Pharmacokinetics. Drugs and the Pharmaceutical Sciences. 2. ed. New York: Marcel Dekker, Inc., 1982. V. 15, p.1-451.
GOHEL, M. C., PANCHAL, M. K. Comparison of in vitro dissolution profiles using a novel model-independent approach. Pharm. Technol., Chester, v.23, p.92-102, 2000 .

GOLDSMITH, J. A., RANDALL, N., ROSS, S. D. On methods of expressing dissolution rate data. J. Pharm. Pharmacol., London, v.30, p.347-349, 1978.

GUIDANCE for industry immediate release solid oral dosage forms scale-up and postapproval changes: chemistry, manufacturing, and controls, in vitro dissolution testing and in vivo bioequivalence documentation, CMC 5. Rockville: Center for Drug Evaluation and Research (CDER), 1995. p.1-25.

HARLAND, R. S., GAZZANIGA, A., SANGALLI, M. E., COLOMBO, P., PEPPAS, N. A. Drug/polymer matrix swelling and dissolution. Pharm. Res., Stuttgart, v.5, p.488-494, 1988.

HIGUCHI, T. Mechanism of sustained-action medication. Theoretical analysis of rate of release of solid drugs dispersed in solid matrices. J. Pharm. Sci., New York, v.52, p.1145-1149, 1963.

HIGUCHI, T. Rate of release of medicaments from ointment bases containing drugs in suspension. J. Pharm. Sci., New York, v.50, p.874-875, 1961.

HIXSON, A. W., CROWELL, J. H. Dependence of reaction velocity upon surface and agitation. Ind. Eng. Chem., London, v.23, p.923-931, 1931.

HOPFENBERG, H. B. Controlled release from erodible slabs, cylinders, and spheres, In: PAUL, D.R., HARRIS, F.W., eds. Controlled Release Polymeric Formulations. Washington: American Chemical Society, 1976. p.26-31. (ACS Symposium Series 33).

HUANG, X., BRAZEL, C. S. On the importance and mechanisms of burst release in matrix-controlled drug delivery systems. J. Control. Release, Amsterdam, v.73, p.121-136, 2001.

JU, H. L., LIAW, S. J. On the assessment of similarity of drug dissolution profiles - A simulation study. Drug Inf. J., Washington, v.31, p.1273-1289, 1997. 
JUN, H. W., LAI, J. W. Preparation and in vitro dissolution tests of egg albumin microcapsules of nitrofurantoin. Int. J. Pharm., Amsterdam, v.16, p.65-77, 1983.

KATZHENDLER, I., HOFMAN, A., GOLDBERGER, A., FRIEDMAN, M. Modeling of drug release from erodible tablets. J. Pharm. Sci., Washington, v.86, p.110-115, 1997.

KHAN, K. A., RHODES, C. T. Effect of compaction pressure on the dissolution efficiency of some direct compression systems. Pharm. Acta Helv., Zurich, v.47, p.594-607, 1972.

KHAN, K. A. The concept of dissolution efficiency. $J$. Pharm. Pharmacol., London, v.27, p.48-49, 1975.

KIM, H., FASSIHI, R. Application of binary polymer system in drug release rate modulation. 2. Influence of formulation variables and hydrodynamic conditions on release kinetics. J. Pharm. Sci., Washington, v.86, p.323328, 1997.

KITAZAWA, S., JOHNO, I., ITO, Y., TERAMURA, S., OKADA, J. Effects of hardness on the disintegration time and the dissolution rate of uncoated caffeine tablets. $J$. Pharm. Pharmacol., London, v.27, p.765-770, 1975.

KITAZAWA, S., JOHNO, I., ITO, Y., TOKUZO, M., OKADA, J. Interpretation of dissolution rate data from in vivo testing of compressed tablets. J. Pharm. Pharmacol., London, v.29, p.453-459, 1977.

KORSMEYER, R. W., GURNY, R., DOELKER, E. M., BURI, P., PEPPAS, N. A. Mechanism of solute release from porous hydrophilic polymers. Int. J. Pharm., Amsterdam, v.15, p.25-35, 1983.

KORSMEYER, R. W., PEPPAS, N.A. Macromolecular and modeling aspects of swelling-controlled systems. In: ROSEMAN, T.J., MANSDORF, S.Z., eds. Controlled release delivery systems. New York: Marcel Dekker, 1981.p.77-90.

LANGENBUCHER, F. Linearization of dissolution rate curves by the Weibull distribution. J. Pharm. Pharmacol., London, v.24, p.979-981, 1972.

LEVENT, K. Data mining exercises for controlled release dosage forms. Pharm. Technol. Europe, Chester, p.1-5, 2001.
LIN, S. Y., YANG, J. C. In-vitro dissolution behavior of some sustained-release theophylline dosage forms, Pharm. Acta Helv., Zurich, v.64, p.236-240, 1989.

LIU, J. P, MA, M. C., CHOW, S. C. Statistical evaluation of Similarity factor $\mathrm{f}_{2}$ as a criterion for assessment of similarity between dissolution profiles. Drug Inf. J., Washington, v.31, p.1255-1271, 1997.

LIU, J. P., CHOW, S. C. Statistical issues on the FDA conjugated estrogen tablets bioequivalence guidance. Drug Inf. J., Washington, v.30, p.881-889, 1996.

MOORE, J. W., FLANNER, H. H. Mathematical comparison of dissolution profiles. Pharm. Technol., Chester, v.20, p.64-74, 1996.

MULYE, N. V., TURCO, S. J. A simple model based on first order kinetics to explain release of highly water soluble drugs from porous dicalcium phosphate dihydrate matrices. Drug Dev. Ind. Pharm., New York, v.21, p.943-953, 1995.

NIEBERGALL, P. J., MILOSOVICH, G., GOYAN, J. E. Dissolution rate studies II: Dissolution of particles under conditions of rapid agitation, J. Pharm. Sci., New York, v.52, p.236-241, 1963.

NOTE for guidance on quality of modified release products: A. Oral dosage forms; B. Transdermal dosage forms; Section I (Quality), CPMP/QWP/604/96. London: The European Agency for the Evaluation of Medicinal Products (EMEA), Human Medicines Evaluation Unit, 1999. p.1-15.

PAIS, J. Intuiting mathematical objects using diagrams and kinetigrams. J. Online Mathematics Applications (JOMA), v.1, 2001.

PEDERSEN, P. V., MYRICK, J. W. Versatile kinetic approach to analysis of dissolution data. J. Pharm. Sci., New York, v.67, p.1450-1455, 1978.

PEPPAS, N. A. Analysis of fickian and non-fickian drug release from polymers. Pharm. Acta Helv., Zurich, v.60, p.110-111, 1985 .

POLLI, J. E., REKHI, G. S., AUGSBURGER, L. L., SHAH, V. P. Methods to compare dissolution profiles and a rationale for wide dissolution specifications for metoprolol tartrate tablets. J. Pharm. Sci., Washington, v.86, p.690-700, 1997. 
PRISTA, L. N., ALVES, A. C., MORGADO, R. M. Técnica farmacêutica e farmácia Galénica. 5. ed. Lisboa: Fundação Calouste Gulbenkian, 1995. v.1, p.453-454.

REPPAS, C., NICOLAIDES, E. Analysis of drug dissolution data. In: DRESSMAN, J. B., LENNERNÄS, eds. Oral drug absorption. Prediction and assessment drugs and the Pharmaceutical Sciences. New York: Marcel Dekker, 2000. v.106, p.229-254.

RESCIGNO, A. Bioequivalence. Pharm. Res., Stuttgart, v.9, p.925-928, 1992.

ROMERO, P., COSTA, J. B., CASTEL-MAROTEAUX, CHULIA, D. Statistical optimization of a controlled release formulation obtained by a double compression process: Application of a Hadamard matrix and a factorial design. In: WELLS, J. I., RUBINSTEIN, M. H., eds. Pharmaceutical technology - Controlled drug release. New York: Ellis Horwood, 1991. v. 2, p.44-58.

SANGALLI, M.E., GIUNCHEDI, P., MAGGI, L., CONTE, U., GAZZANIGA, A. Inert monolithic device with a central hole for constant drug release. Eur. J. Pharm. Biopharm., Amsterdam, v.40, p.370-373, 1994.

SARANADASA, H. Defining similarity of dissolution profiles through Hotelling's T2 statistic. Pharm. Technol., Chester, v.25, p.46-54, 2001.

SCHWARTZ, B. J., SIMONELLI, A. P., HIGUCHI, W. I. Drug release from wax matrices I - Analysis of data with first-order kinetics and with the diffusion-controlled model. J. Pharm. Sci., New York, v.57, p.274-277, 1968.

SCHWARTZ, B. J., SIMONELLI, A. P., HIGUCHI, W. I. Drug release from wax matrices II - application of a mixture theory to the sulfanilamide-wax system. $J$. Pharm. Sci., New York, v.57, p.278-282, 1968.

SEKI, T., KAWAGUCHI, T., ENDOH, H. ISHIKAWA, K., JUNI, K., NAKANO, M. Controlled release of 3',5'diester prodrugs of 5-fluoro-2'-deoxyuridine from poly-L-lactic acid microspheres. J. Pharm. Sci., New York, v.79, p.985-987, 1980.
SHAH, V. P., POLLI, J. E. Methods to compare dissolution profiles. Drug Inf. J., Washington, v.30, p.1113-1120, 1996.

SHAH, V. P., TSONG, Y., SATHE, P., LIU, J. P. In vitro dissolution profile comparison. Statistics and analysis of the similarity factor, $\mathrm{f}_{2}$. Pharm. Res., Stuttgart, v.15, p.889-896, 1998.

SHUKLA, A. J., PRICE, J. C. Effect of drug (core) particle size on the dissolution of theophylline from microspheres made from low molecular weight cellulose acetate propionate. Pharm. Res., Stuttgart, v.6, p.418-421, 1989.

SHUKLA, A. J., PRICE, J. C. Effect of drug loading and molecular weight of cellulose acetate propionate on the release characteristics of theophylline microspheres. Pharm. Res., Stuttgart, v.8, p.1369-1400, 1991.

TSONG, Y., HAMMERSTROM, T., SATHE, P., SHAH, V. P., Statistical assessment of mean differences between two dissolution data sets. Drug Inf. J., Washington, v.30, p.1105-1112, 1996.

VARELAS, C. G., DIXON, D. G., CAROL, S. Zero-order release from biphasic polymer hydrogels. J. Control. Release, Amsterdam, v.34, p.185-192, 1995.

VUDATHALA, G. K., ROGERS, J. A. Dissolution of fludrocortisone from phospholipid coprecipitates. $J$. Pharm. Sci., Washington, v.82, p.282-286, 1992.

WAGNER, J. G. Interpretation of percent dissolved-time plots derived from In vitro testing of conventional tablets and capsules. J. Pharm. Sci., New York, v.58, p.12531257, 1969.

Recebido para publicação em 04 de outubro de 2001. 